\title{
GENERALIZED HILBERT FUNCTIONS
}

\author{
CLAUDIA POLINI AND YU XIE
}

\begin{abstract}
Let $M$ be a finite module and let $I$ be an arbitrary ideal over a Noetherian local ring. We define the generalized Hilbert function of $I$ on $M$ using the 0th local cohomology functor. We show that our definition re-conciliates with that of Ciupercă. By generalizing Singh's formula (which holds in the case of $\lambda(M / I M)<\infty)$, we prove that the generalized Hilbert coefficients $j_{0}, \ldots, j_{d-2}$ are preserved under a general hyperplane section, where $d=\operatorname{dim} M$. We also keep track of the behavior of $j_{d-1}$. Then we apply these results to study the generalized Hilbert function for ideals that have minimal $j$-multiplicity or almost minimal $j$-multiplicity. We provide counterexamples to show that the generalized Hilbert series of ideals having minimal or almost minimal $j$-multiplicity does not have the 'expected' shape described in the case where $\lambda(M / I M)<\infty$. Finally we give a sufficient condition such that the generalized Hilbert series has the desired shape.
\end{abstract}

\section{INTRODUCTION}

In this paper we define the generalized Hilbert function of $I$, where $I$ is an arbitrary ideal over a Noetherian local ring $(R, \mathfrak{m})$. We study its behavior under general hyperplane sections and investigate the interplay between the generalized Hilbert function and the depth of the associated graded ring. The associated graded ring $\operatorname{gr}_{I}(R):=\oplus_{t=1}^{\infty} I^{t} / I^{t+1}$ of $I$ is an algebraic construction whose projective scheme represents the exceptional fiber of the blowup of a variety along a subvariety. The Hilbert function of $I$ provides useful information on its arithmetical properties, like its depth, which in turn give information, for instance, on the cohomology groups of the blowup.

In this introduction we will only discuss the case of associated graded rings, although in the rest of the paper we will treat associated graded modules.

For an $\mathfrak{m}$-primary ideal $I$, the Hilbert-Samuel function is defined to be the numerical function $H_{I}(t)$ that measures the growth of the length $\lambda\left(R / I^{t+1}\right)$ of the $(t+1)$ th power of $I$ for all $t \geq 0$. For $t$ sufficiently large, the function $H_{I}(t)$ is a polynomial in $t$ of degree $d$, the dimension of $R$. This is the Hilbert-Samuel polynomial $P_{I}(t)$ of $I$, whose normalized coefficients $e_{i}(I), 0 \leq i \leq d$, dubbed the Hilbert-Samuel coefficients of $I$, are uniquely determined by $I$. The notion of Hilbert function is fundamental and has been widely studied by algebraists and geometers. For example, the well-known Singh's formula proved for the Hilbert-Samuel function yields the fact that the HilbertSamuel coefficients $e_{0}, \ldots, e_{d-2}$ are preserved under a general hyperplane section. It also allows us to keep track of the behaviors of $e_{d-1}$ and $e_{d}$ under a general hyperplane section.

The interplay between the Hilbert function of $I$, more precisely its Hilbert coefficients, and the depth of the associated graded ring has been widely investigated. This line of study has its roots

AMS 2010 Mathematics Subject Classification. Primary 13D40 and 13A30; Secondary 13H15.

The first author was partially supported by the NSF and the NSA. 
in the pioneering work of Sally. The idea is that extremal values of the Hilbert coefficients, most notably of the multiplicity of $I$, yield high depth of the associated graded ring and, conversely, good depth properties encode all the information about the Hilbert function. In 1967 Abhyankar proved that the multiplicity $e(R)=e_{0}(\mathfrak{m})$ of a $d$-dimensional Cohen-Macaulay local ring is bounded below by $\mu(\mathfrak{m})-d+1$, where $\mu(\mathfrak{m})$ is the embedding dimension of $R$ [1]. Rings for which $e_{0}=\mu(\mathfrak{m})-$ $d+1$ have since then been called rings of minimal multiplicity. In the case of minimal multiplicity, Sally had shown that the associated graded ring $\operatorname{gr}_{\mathfrak{m}}(R)$ is always Cohen-Macaulay with the HilbertSamuel series $h_{I}(z):=\sum_{t=0}^{\infty} H_{I}(t) z^{t}=(1+h z) /(1-z)^{d+1}$, where $h=\mu(\mathfrak{m})-d$ is the embedding codimension of $R$ [19]. Almost twenty years later, Rossi-Valla [15] and Wang [24] independently proved that if the multiplicity of $R$ is almost minimal then the depth $\operatorname{of}_{\mathfrak{m}}(R)$ is almost maximal, i.e., it is at least $d-1$. Furthermore in [15], all the possible Hilbert-Samuel series of rings of almost minimal multiplicity have been described to be of the form $h_{I}(z)=\left(1+h z+z^{s}\right) /(1-z)^{d+1}$, where $2 \leq s \leq h+1$. Since then there have been many generalizations of these results to $\mathfrak{m}$-primary ideals and to modules having ideal filtrations of finite colength, a condition that is required to define the classical Hilbert function (see for example [16], [8], [6], [7], [18], [14], [17]).

The first work to generalize the theory of minimal and almost minimal multiplicity to arbitrary ideals was done by Polini and Xie [13]. If the ideal $I$ is not $\mathfrak{m}$-primary then the Hilbert function is not defined, thus there is no numerical information on Hilbert coefficients available to study the Cohen-Macaulayness of $\operatorname{gr}_{I}(R)$. To remedy the lack of this tool, Polini and Xie proposed to use the notion of $j$-multiplicity. The $j$-multiplicity was introduced by Achilles and Manaresi [2] in 1993 as a generalization of the Hilbert multiplicity to arbitrary ideals. It has been frequently used by both algebraists and geometers as an invariant to deal with improper intersections and non-isolated singularities [2].

In [13], Polini and Xie proved a lower bound for the $j$-multiplicity of an ideal and then gave the definition of ideals having minimal $j$-multiplicity or almost minimal $j$-multiplicity. Under certain residual conditions, they proved that for ideals having minimal $j$-multiplicity (respectively, almost minimal $j$-multiplicity), the associated graded ring is Cohen-Macaulay (respectively, almost CohenMacaulay). Their work has been extended further very recently by Mantero and Xie by introducing the concept of $j$-stretched ideals [10]. Although both work successfully generalized many classical results, there is no description of the Hilbert function as it has been done for m-primary ideals.

In this paper, we define the generalized Hilbert function of an arbitrary ideal $I$ on a finite module $M$ over a Noetherian local ring using 0th local cohomology. We then present Ciupercă's approach and re-conciliate that with ours. We generalize Singh's formula to arbitrary ideals and show that the first $d-1$ generalized Hilbert coefficients $j_{0}, \ldots, j_{d-2}$ are preserved under a general hyperplane section, where $d$ is the dimension of the module $M$. Furthermore, we keep track of how the next generalized Hilbert coefficient $j_{d-1}$ changes. In Section 3, we study the generalized Hilbert function for ideals that have minimal $j$-multiplicity or have almost minimal $j$-multiplicity on $M$. We provide counterexamples to show that the generalized Hilbert series of ideals having minimal or almost 
minimal $j$-multiplicity on $M$ does not have the 'expected' shape as described in the case where $\lambda(M / I M)<\infty$. We then give a sufficient condition such that the generalized Hilbert series has the desired shape.

The study of the generalized Hilbert function is of independent interest and for example will be used in a future work where we investigate the first generalized Hilbert coefficient $j_{1}$ in relation with the normalization of the Rees algebra [11].

\section{BASIC PROPERTIES OF GENERALIZED HILBERT FUNCTIONS}

Let $M$ be a finite module over a Noetherian local ring $(R, \mathfrak{m})$ and $I$ an arbitrary $R$-ideal. Let $T=\operatorname{gr}_{I}(M):=\oplus_{t=0}^{\infty} I^{t} M / I^{t+1} M$ be the associated graded module of $I$ on $M$. Observe $T$ is a finite graded module over the associated graded ring $G=\operatorname{gr}_{I}(R):=\oplus_{t=0}^{\infty} I^{t} / I^{t+1}$. In general the homogeneous components of $T$ may not have finite length, thus we consider the $T$-submodule of elements supported on $\mathfrak{m}, W=\Gamma_{\mathfrak{m}}(T)=0:_{T} \mathfrak{m}^{\infty}=\oplus_{t=0}^{\infty} \Gamma_{\mathfrak{m}}\left(I^{t} M / I^{t+1} M\right)$. Since $W$ is annihilated by a large power of $\mathfrak{m}$, it is a finite graded module over $\operatorname{gr}_{I}(R) \otimes_{R} R / \mathfrak{m}^{\alpha}$ for some $\alpha \geq 0$, hence its Hilbert-Samuel function $H_{W}(t)=\sum_{v=0}^{t} \lambda\left(\Gamma_{\mathfrak{m}}\left(I^{v} M / I^{v+1} M\right)\right)$ is well defined. We define the generalized Hilbert-Samuel function of $I$ on $M: H_{I, M}(t):=H_{W}(t)$ for every $t \geq 0$, and the generalized Hilbert-Samuel series of $I$ on $M: h_{I, M}(z):=\sum_{t=0}^{\infty} H_{W}(t) z^{t}$. By the Hilbert-Serre theorem, we know that the series is of the form

$$
h_{I, M}(z)=h(z) /(1-z)^{r+1},
$$

where $h(z) \in \mathbb{Z}[z], h(1) \neq 0$ and $r=\operatorname{dim}_{G} W$. The polynomial $h(z)$ is called the $h$-polynomial of $I$ on $M$.

Let $\operatorname{dim} M=d$. Then $\operatorname{dim}_{G} W \leq \operatorname{dim}_{G} T=d$, thus $H_{I, M}(t)$ is eventually a polynomial

$$
P_{I, M}(t)=\sum_{i=0}^{d}(-1)^{i} j_{i}(I, M)\left(\begin{array}{c}
t+d-i \\
d-i
\end{array}\right)
$$

of degree at most $d$. We define $P_{I, M}(t)$ to be the generalized Hilbert-Samuel polynomial of $I$ on $M$ and $j_{i}(I, M), 0 \leq i \leq d$, the generalized Hilbert-Samuel coefficients of $I$ on $M$. Observe that the normalized leading coefficient $j_{0}(I, M)$ is also called the $j$-multiplicity of $I$ on $M$ (see for instance [2] or [12]).

Recall that the Krull dimension of the special fiber module $T / \mathfrak{m} T$ is called the analytic spread of $I$ on $M$ and is denoted by $\ell(I, M)$. In general, $\operatorname{dim}_{G} W \leq \ell(I, M) \leq d$ and equalities hold if and only if $\ell(I, M)=d$. Therefore $j_{0}(I, M) \neq 0$ if and only if $\ell(I, M)=d[12,2.1]$.

If $M / I M$ has finite length, the ideal $I$ is said to be an ideal of definition on $M$. In this case each homogeneous component of $T$ has finite length, thus $W=T$ and the generalized HilbertSamuel function coincides with the usual Hilbert-Samuel function; in particular the generalized Hilbert-Samuel coefficients $j_{i}(I, M)$ coincides with the usual Hilbert-Samuel coefficients $e_{i}(I, M)$, $0 \leq i \leq d$.

The definition of generalized Hilbert function, generalized Hilbert polynomial, and corresponding generalized Hilbert coefficients is new to the literature. It appeared first in an unpublished work 
of Polini and Ulrich. It stems from the original definition of $j$-multiplicity given by Achilles and Manaresi [2]. The definition of higher generalized Hilbert coefficients presented in the literature is quite different (see [3] and [4]). We review now Ciupercă's approach and re-conciliate that with ours. More precisely, for each $i$, Ciupercă defined a sequence of numbers which represents the $i$ th generalized Hilbert coefficient [4, 2.1]. We will show that our $(-1)^{i} j_{i}(I, M)$ is the last element in that sequence (see Proposition 2.4).

Let $q$ be an ideal of definition on $M / I M$. We form the bigraded module $T^{\prime}=\operatorname{gr}_{q}\left(\operatorname{gr}_{I}(M)\right)=$ $\oplus_{s, t=0}^{\infty}\left(q^{s} I^{t} M+I^{t+1} M\right) /\left(q^{s+1} I^{t} M+I^{t+1} M\right)$, then $T^{\prime}$ is a finite bigraded module over the bigraded ring $G^{\prime}=\oplus_{s, t=0}^{\infty}\left(q^{s} I^{t}+I^{t+1}\right) /\left(q^{s+1} I^{t}+I^{t+1}\right)$. Notice that every homogeneous component of $T^{\prime}$ has finite length. Let

$$
\begin{gathered}
H_{(q, I, M)}^{(0,0)}(s, t)=\lambda\left(\left(q^{s} I^{t} M+I^{t+1} M\right) /\left(q^{s+1} I^{t} M+I^{t+1} M\right)\right) \\
H_{(q, I, M)}^{(1,0)}(s, t)=\sum_{\mu=0}^{s} H_{(q, I, M)}^{(0,0)}(\mu, t)=\lambda\left(I^{t} M /\left(q^{s+1} I^{t} M+I^{t+1} M\right)\right),
\end{gathered}
$$

and

$$
H_{(q, I, M)}^{(1,1)}(s, t)=\sum_{v=0}^{t} H_{(q, I, M)}^{(1,0)}(s, v)=\lambda\left(M /\left(q^{s+1} M+I M\right)\right)+\cdots+\lambda\left(I^{t} M /\left(q^{s+1} I^{t} M+I^{t+1} M\right)\right) .
$$

For $s$ and $t$ sufficiently large, $H_{(q, I, M)}^{(0,0)}(s, t), H_{(q, I, M)}^{(1,0)}(s, t)$ and $H_{(q, I, M)}^{(1,1)}(s, t)$ are polynomials $P_{(q, I, M)}^{(0,0)}(s, t)$, $P_{(q, I, M)}^{(1,0)}(s, t)$ and $P_{(q, I, M)}^{(1,1)}(s, t)$ in two variables $s, t$ of degree at most $d-2, d-1$ and $d$ respectively. One can write

$$
\begin{aligned}
& P_{(q, I, M)}^{(0,0)}(s, t)=\sum_{i, j \geq 0, i+j \leq d-2} a_{(q, I, M)}^{(0,0)}(i, j)\left(\begin{array}{c}
s+i \\
i
\end{array}\right)\left(\begin{array}{c}
t+j \\
j
\end{array}\right), \\
& P_{(q, I, M)}^{(1,0)}(s, t)=\sum_{i, j \geq 0, i+j \leq d-1} a_{(q, I, M)}^{(1,0)}(i, j)\left(\begin{array}{c}
s+i \\
i
\end{array}\right)\left(\begin{array}{c}
t+j \\
j
\end{array}\right),
\end{aligned}
$$

and

$$
P_{(q, I, M)}^{(1,1)}(s, t)=\sum_{i, j \geq 0, i+j \leq d} a_{(q, I, M)}^{(1,1)}(i, j)\left(\begin{array}{c}
s+i \\
i
\end{array}\right)\left(\begin{array}{c}
t+j \\
j
\end{array}\right) .
$$

Then $a_{(q, I, M)}^{(0,0)}(i, j)=a_{(q, I, M)}^{(1,0)}(i+1, j)$ for $i, j \geq 0, i+j \leq d-2$ and $a_{(q, I, M)}^{(1,0)}(i, j)=a_{(q, I, M)}^{(1,1)}(i, j+1)$ for $i, j \geq 0, i+j \leq d-1$. The $i$ th generalized Hilbert coefficient defined by Ciupercă is as follows [4, 2.1]:

$$
j_{i}(q, I, M)=\left(a_{(q, I, M)}^{(1,1)}(i, d-i), a_{(q, I, M)}^{(1,1)}(i-1, d-i), \ldots, a_{(q, I, M)}^{(1,1)}(0, d-i)\right) \in \mathbb{Z}^{i+1}
$$

for every $0 \leq i \leq d$.

Notice that $j_{0}(q, I, M)=a_{(q, I, M)}^{(1,1)}(0, d)=a_{(q, I, M)}^{(1,0)}(0, d-1)$ and $a_{(q, I, M)}^{(1,1)}(0, d)=j_{0}(I, M)$ the $j$ multiplicity of $I$ on $M$ (see [3] or [4]). Moreover, if $I$ is an ideal of definition on $M$, then for $0 \leq i \leq d, a_{(q, I, M)}^{(1,1)}(0, d-i)=(-1)^{i} j_{i}(I, M)=(-1)^{i} e_{i}(I, M)$ and $a_{(q, I, M)}^{(1,1)}(l, d-i)=0$ if $0<l \leq i$ ([3], [4]). In particular, $a_{(q, I, M)}^{(1,1)}(0, d)=j_{0}(I, M)=e_{0}(I, M)$ is the usual Hilbert-Samuel multiplicity 
of $I$ on $M$. Our first goal is to show that for any ideal $I$, the coefficient $a_{(q, I, M)}^{(1,1)}(0, d-i)$ coincides with $(-1)^{i} j_{i}(I, M)$ for suitable choice of $q$ (see Proposition 2.4).

In the following lemma (Lemma2.2) we are going to show that Ciupercă's $i$ th generalized Hilbert coefficient behaves well under a general hyperplane section for $0 \leq i \leq d-2$. This generalizes Proposition 2.11 in [4] since we do not assume that the hyperplane section is a nonzero divisor. Furthermore, we keep track of how the $(d-1)$ th generalized Hilbert coefficient $j_{d-1}(q, I, M)$ changes (notice that there is an error in [4, 2.11], namely $j_{d-1}(q, I, M)$ is not preserved, see Example ??). The proof uses a generalization of Singh's formula (see Lemma 2.1) as it is done in the case of ideals of definition on $M$ (see [17, Lemma 1.6, Proposition 1.2]).

Lemma 2.1. Let $R$ be a Noetherian local ring, let I be any $R$-ideal, and let $M$ be a finitely generated $R$-module. For any $x \in I$ write $\bar{M}=M / x M$. The generalized Singh's formula holds for any non negative integers $s$ and $t$ :

$$
\begin{aligned}
& H_{(q, I, M)}^{(1,0)}(s, t)=H_{(q, I, \bar{M})}^{(1,1)}(s, t) \\
& +\sum_{v=2}^{t} \lambda\left(I^{v} M:_{M} x /\left[\left(q^{s+1} I^{v} M+I^{v+1} M\right):_{M} x+I^{v-1} M\right]\right) \\
& -\sum_{v=1}^{t} \lambda\left(\left[\left(q^{s+1} I^{v} M+I^{v+1} M\right):_{I^{v-1} M} x\right] /\left[q^{s+1} I^{v-1} M+I^{v} M\right]\right) .
\end{aligned}
$$

Moreover if I is an ideal of definition on $M$, then for $s>>0$, the above formula becomes the usual Singh's formula:

$$
\lambda\left(I^{t} M / I^{t+1} M\right)=\lambda\left(M / I^{t+1} M+x M\right)-\lambda\left(I^{t+1} M:_{M} x / I^{t} M\right) .
$$

Proof. First, for every $v \geq 0$, there is an exact sequence:

$$
\begin{gathered}
0 \longrightarrow\left[\left(q^{s+1} I^{v} M+I^{v+1} M\right):_{I^{v-1} M} x\right] /\left[q^{s+1} I^{\nu-1} M+I^{v} M\right] \longrightarrow \\
I^{v-1} M /\left[q^{s+1} I^{\nu-1} M+I^{v} M\right] \stackrel{x}{\longrightarrow} I^{v} M /\left[q^{s+1} I^{\nu} M+I^{\nu+1} M\right] \\
\longrightarrow I^{v} M /\left[x I^{v-1} M+q^{s+1} I^{v} M+I^{v+1} M\right] \longrightarrow 0 .
\end{gathered}
$$

By adding $\mathrm{v}$ up to $t$, we obtain that

$$
\begin{aligned}
& H_{(q, I, M)}^{(1,0)}(s, t)=H_{(q, I, \bar{M})}^{(1,1)}(s, t) \\
& +\sum_{v=2}^{t} \lambda\left(\left[x M \cap I^{v} M+q^{s+1} I^{v} M+I^{v+1} M\right] /\left[x I^{v-1} M+q^{s+1} I^{v} M+I^{v+1} M\right]\right) \\
& -\sum_{v=1}^{t} \lambda\left(\left[\left(q^{s+1} I^{v} M+I^{v+1} M\right):_{I^{v-1} M} x\right] /\left[q^{s+1} I^{v-1} M+I^{v} M\right]\right) .
\end{aligned}
$$

Observe that

$$
\begin{aligned}
& {\left[x M \cap I^{v} M+q^{s+1} I^{v} M+I^{v+1} M\right] /\left[x I^{\nu-1} M+q^{s+1} I^{v} M+I^{v+1} M\right]} \\
& \cong x M \cap I^{v} M /\left[x M \cap\left(q^{s+1} I^{v} M+I^{v+1} M\right)+x I^{\nu-1} M\right] \\
& =x\left(I^{v} M:_{M} x\right) / x\left[\left(q^{s+1} I^{\nu} M+I^{\nu+1} M\right):_{M} x+I^{\nu-1} M\right] .
\end{aligned}
$$


It is easy to see that the surjective map $I^{v} M:_{M} x \stackrel{x}{\rightarrow} x\left(I^{v} M:_{M} x\right) / x\left[\left(q^{s+1} I^{v} M+I^{v+1} M\right):_{M} x+I^{v-1} M\right]$ has kernel $\left(q^{s+1} I^{v} M+I^{v+1} M\right):_{M} x+I^{v-1} M$. Therefore

$$
\begin{aligned}
& \lambda\left(\left[x M \cap I^{v} M+q^{s+1} I^{v} M+I^{v+1} M\right] /\left[x I^{v-1} M+q^{s+1} I^{v} M+I^{v+1} M\right]\right) \\
& =\lambda\left(x\left(I^{v} M:_{M} x\right) / x\left[\left(q^{s+1} I^{v} M+I^{v+1} M\right):_{M} x+I^{v-1} M\right]\right) \\
& =\lambda\left(I^{v} M:_{M} x /\left[\left(q^{s+1} I^{v} M+I^{v+1} M\right):_{M} x+I^{\nu-1} M\right]\right) .
\end{aligned}
$$

By plugging in equation (1) we obtain the desired formula.

For the second part, observe that if $I$ is an ideal of definition on $M$ then for $s>>0, q^{s+1} M \subseteq I M$. Hence the formula in the statement of Lemma 2.1 becomes

$$
\begin{aligned}
& \lambda\left(I^{t} M / I^{t+1} M\right)-\lambda\left(M / I^{t+1} M+x M\right) \\
& =\sum_{v=2}^{t} \lambda\left(I^{v} M:_{M} x /\left[I^{v+1} M:_{M} x+I^{\nu-1} M\right]\right)-\sum_{v=1}^{t} \lambda\left(I^{\nu+1} M:_{I^{v-1} M} x / I^{v} M\right) \\
& =\sum_{v=2}^{t} \lambda\left(I^{\nu} M:_{M} x / I^{\nu-1} M\right)-\sum_{\nu=2}^{t} \lambda\left(\left[I^{\nu+1} M:_{M} x+I^{\nu-1} M\right] / I^{\nu-1} M\right)-\sum_{\nu=1}^{t} \lambda\left(I^{\nu+1} M:_{\Gamma^{\nu-1}} x x / I^{\nu} M\right) \\
& =\sum_{v=2}^{t} \lambda\left(I^{\nu} M:_{M} x / I^{\nu-1} M\right)-\sum_{v=2}^{t} \lambda\left(I^{\nu+1} M:_{M} x / I^{\nu+1} M:_{\Gamma^{\nu-1}} x\right)-\sum_{v=1}^{t} \lambda\left(I^{\nu+1} M:_{\Gamma^{\nu-1} M} x / I^{\nu} M\right) \\
& =\sum_{v=2}^{t} \lambda\left(I^{v} M:_{M} x / I^{\nu-1} M\right)-\sum_{v=1}^{t} \lambda\left(I^{\nu+1} M:_{M} x / I^{v} M\right) \\
& =-\lambda\left(I^{t+1} M:_{M} x / I^{t} M\right) \text {. }
\end{aligned}
$$

We now need to recall some definitions and basic facts (see for instance [2], [9], [21] and [13]). Let $(R, \mathfrak{m})$ be a Noetherian local ring, $I$ an $R$-ideal, $M$ a finite $R$-module, and $G$ and $T$ are defined as before. An element $x \in R$ is said to be a filter-regular element for $M$ with respect to $I$, if $0: M$ $x \subseteq 0: M I^{\infty}$. This holds if and only if $x$ avoids all associated primes of $M$ that do not contain $I$. A sequence of elements $x_{1}, \ldots, x_{l}$ of $I$ is called a filter-regular sequence for $M$ with respect to $I$, if $\left(x_{1}, \ldots, x_{i-1}\right) M:_{M} x_{i} \subseteq\left(x_{1}, \ldots, x_{i-1}\right) M:_{M} I^{\infty}$ for $1 \leq i \leq l$. The sequence $x_{1}, \ldots, x_{l}$ of $I$ is called a superficial sequence for $M$ with respect to $I$ if the initial forms $x_{1}^{*}, \ldots, x_{l}^{*}$ of $x_{1}, \ldots, x_{l}$ in $G$ are of degree one and form a filter-regular sequence for $T$ with respect to $G_{+}$, where $G_{+}$is the ideal generated by all homogeneous elements of positive degree in $G$. It is well-known that if $x_{1}, \ldots, x_{l}$ form a superficial sequence then they also form a filter-regular sequence.

Let $I=\left(a_{1}, \ldots, a_{n}\right)$. Recall that $a_{1}, \ldots, a_{n}$ form a $d$-sequence on $M$ (or an absolutely superficial $M$-sequence in the sense of Trung) if $\left[\left(a_{1}, \ldots, a_{i-1}\right) M:_{M} a_{i}\right] \cap I M=\left(a_{1}, \ldots, a_{i-1}\right) M$ for $1 \leq i \leq n$.

Let $I=\left(a_{1}, \ldots, a_{n}\right)$ and write $x_{i}=\sum_{j=1}^{n} \lambda_{i j} a_{j}$ for $1 \leq i \leq l$ and $\left(\lambda_{i j}\right) \in R^{l n}$. The elements $x_{1}, \ldots, x_{l}$ form a sequence of general elements in $I$ (equivalently $x_{1}, \ldots, x_{l}$ are general in $I$ ) if there exists a dense open subset $U$ of $K^{l n}$ such that the image $\left(\overline{\lambda_{i j}}\right) \in U$, where $K=R / \mathfrak{m}$ is the residual field of $R$. When $l=1, x=x_{1}$ is said to be general in $I$. 
The notion of general elements is a fundamental tool for our study as they are always a superficial sequence for $M$ with respect to $I[25,2.5]$; they generate a minimal reduction $J$ whose reduction number $r_{J}(I, M)$ coincides with the reduction number $r(I, M)$ of $I$ on $M$ if $l=\ell(I, M)$ (see [22, 2.2] and [20,8.6.6]); and they form a super-reduction in the sense of [2] whenever $l=\ell(I, M)=d=$ $\operatorname{dim}_{R} M$ (see [25, 2.5]). Furthermore, one can compute the $j$-multiplicity using general elements.

The next proposition states the behaviors of Ciupercă's generalized Hilbert coefficients under a general hyperplane section.

Proposition 2.2. Let $R$ be a Noetherian local ring with infinite residue field, let I be any $R$-ideal, and let $M$ be a finitely generated R-module of dimension $d$. If $x$ is a general element in I then the following statements hold:

(a) $j_{i}(q, I, M)=j_{i}(q, I, M / x M)$ for $0 \leq i \leq d-2$.

(b) There exists a fixed positive integer $t_{0}$ such that if $t>t_{0}$,

$$
\begin{gathered}
\sum_{v=2}^{t} \lambda\left(I^{v} M:_{M} x /\left[\left(q^{s+1} I^{v} M+I^{\nu+1} M\right):_{M} x+I^{v-1} M\right]\right) \\
-\sum_{v=1}^{t} \lambda\left(\left[\left(q^{s+1} I^{v} M+I^{\nu+1} M\right):_{I^{v-1} M} x\right] /\left[q^{s+1} I^{\nu-1} M+I^{v} M\right]\right)
\end{gathered}
$$

is a polynomial $\sum_{i=0}^{d-1} \beta_{i}\left(\begin{array}{c}s+i \\ i\end{array}\right)$ in s of degree at most $d-1$ (i.e., it does not depend on $t$ ). Therefore $j_{d-1}(q, I, M)=j_{d-1}(q, I, M / x M)+\left(\beta_{d-1}, \ldots, \beta_{0}\right)$.

Proof. By avoiding finitely many associated prime ideals of $T^{\prime}$ which do not contain $I / q I$, we obtain a general element $x$ in $I$ such that the image $x^{\prime}$ in $I / q I$ is filter-regular for $T^{\prime}$ with respect to the ideal $(I / q I) G^{\prime}$. Thus there exists $t_{0}>0$ such that for every $t>t_{0}$ and for every $s \geq 0, x M \cap I^{t} M=x I^{t-1} M$ and $\left(q^{s+1} I^{t} M+I^{t+1} M\right): I_{I^{t-1} M} x=q^{s+1} I^{t-1} M+I^{t} M$. Write $\bar{M}=M / x M$. By Lemma 2.1 we obtain for sufficiently large $s$ and $t$,

$$
\begin{aligned}
P_{(q, I, M)}^{(1,0)}(s, t)=P_{(q, I, \bar{M})}^{(1,1)}(s, t) & +\sum_{v=2}^{t_{0}} \lambda\left(I^{\nu} M:_{M} x /\left[\left(q^{s+1} I^{\nu} M+I^{\nu+1} M\right):_{M} x+I^{\nu-1} M\right]\right) \\
& -\sum_{v=1}^{t_{0}} \lambda\left(\left[\left(q^{s+1} I^{\nu} M+I^{\nu+1} M\right):_{I^{v-1} M} x\right] /\left[q^{s+1} I^{\nu-1} M+I^{v} M\right]\right) .
\end{aligned}
$$

Now the desired equality follows from (2) by equaling the coefficients.

The following lemma follows from [21, 1.1, 2.1].

Lemma 2.3. Let $(R, \mathfrak{m})$ be a Noetherian local ring with infinite residue field, let $G$ be a standard graded ring over $R$, and let $T$ be a finite G-module. Fix a positive integer $l$. Then there exists $\underline{y}=y_{1}, \ldots, y_{l}$ in $\mathfrak{m}$ such that $\underline{y}$ forms a d-sequence on $T$, i.e., for every $1 \leq i \leq l$,

$$
\left(y_{1}, \ldots, y_{i-1}\right) T:_{T} y_{i}=\left(y_{1}, \ldots, y_{i-1}\right) T:_{T} \mathfrak{m}^{\infty}
$$


and

$$
\left[\left(y_{1}, \ldots, y_{i-1}\right) T:_{T} y_{i}\right] \cap\left(y_{1}, \ldots, y_{l}\right) T=\left(y_{1}, \ldots, y_{i-1}\right) T .
$$

In particular, if $T=\oplus_{t=0}^{\infty} T_{t}$ is a finite graded module over $G$, then for every $t \geq 0$,

$$
\left(y_{1}, \ldots, y_{i-1}\right) T_{t}:_{T_{t}} y_{i}=\left(y_{1}, \ldots, y_{i-1}\right) T_{t}:_{T_{t}} \mathfrak{m}^{\infty}
$$

and

$$
\left[\left(y_{1}, \ldots, y_{i-1}\right) T_{t}:_{t} y_{i}\right] \cap\left(y_{1}, \ldots, y_{l}\right) T_{t}=\left(y_{1}, \ldots, y_{i-1}\right) T_{t}
$$

which shows that $\underline{y}$ forms an absolutely superficial $T_{t}$-sequence for every $t \geq 0$.

Proof. Since $T$ is a finite $G$-module and $\mathfrak{m} G$ is a $G$-ideal, there exist $a_{1}, \ldots, a_{l}$ in $\mathfrak{m}$ such that their initial forms $a_{1}^{*}, \ldots, a_{l}^{*}$ in $\mathfrak{m} G$ form a filter-regular sequence for $T$ with respect to $\mathfrak{m} G$. By [21, 2.1], there is an ascending sequence of integers $n_{1} \leq \mathfrak{n}_{2} \leq \ldots \leq \mathfrak{n}_{l}$ such that $\left(a_{1}^{*}\right)^{n_{1}}, \ldots,\left(a_{l}^{*}\right)^{n_{l}}$ form a $d$-sequence on $T$. Set $\underline{y}=a_{1}^{n_{1}}, \ldots, a_{l}^{n_{l}}$. Then the conclusions follow from [21, 1.1].

In the following proposition we achieve our first goal. We show that how our generalized Hilbert coefficients are related to the generalized Hilbert coefficients of Ciupercă. The proof of this result follows essentially from Lemma 2.3 and [21, 4.1].

Proposition 2.4. Let $(R, \mathfrak{m})$ be a Noetherian local ring with infinite residue field, let $I$ be any $R$ ideal, let $M$ be a finitely generated R-module of dimension $d$, and let $T$ be the associated graded module of I on $M$. Then there exist elements $y_{1}, \ldots, y_{d} \in \mathfrak{m}$ which form a $d$-sequence on $T_{t}$ for every $t \geq 0$. Therefore if we set $q=\left(y_{1}, \ldots, y_{d}\right)$ then $a_{(q, I, M)}^{(1,1)}(0, d-i)=(-1)^{i} j_{i}(I, M)$ for every $0 \leq i \leq d$.

Proof. By Lemma 2.3, there exist elements $y_{1}, \ldots, y_{d} \in \mathfrak{m}$ such that $y_{1}, \ldots, y_{d}$ form a $d$-sequence on $T_{t}$ for every $t \geq 0$, in particular, they form a system of parameters for $T_{t}$. By [21, 4.1], for every $t \geq 0$,

$$
H_{T^{\prime}}^{(1,0)}(s, t)=\lambda\left(T_{t} / q^{s+1} T_{t}\right)=\sum_{i=0}^{d}\left[\lambda\left(q_{i} T_{t}: T_{t} \mathfrak{m}^{\infty} / q_{i} T_{t}\right)-\lambda\left(q_{i-1} T_{t}: T_{t} \mathfrak{m}^{\infty} / q_{i-1} T_{t}\right)\right]\left(\begin{array}{c}
s+i \\
i
\end{array}\right),
$$

where $q_{i}=\left(y_{1}, \ldots, y_{i}\right), 1 \leq i \leq d, q_{0}=(0)$ and $q_{-1} T_{t}:_{T_{t}} \mathfrak{m}^{\infty} / q_{-1} T_{t}=(0) T_{t}$.

Thus for $t>>0$,

$$
\begin{aligned}
\sum_{i=0}^{d}(-1)^{i} j_{i}(I, M)\left(\begin{array}{c}
t+d-i \\
d-i
\end{array}\right) & =\sum_{v=0}^{t} \lambda\left(0:_{T_{v}} \mathfrak{m}^{\infty}\right) \\
& =\sum_{i=0}^{d} a_{(q, I, M)}^{(1,1)}(0, d-i)\left(\begin{array}{c}
t+d-i \\
d-i
\end{array}\right) .
\end{aligned}
$$

We are done by equaling corresponding coefficients.

We now recall the notion of specialization from [23]. Let $S=V[\underline{x}]$ be a polynomial ring over a regular local ring $(V, \mathfrak{n})$ and $\widetilde{S}=V[\underline{z}]_{\mathfrak{n} V[z]}[\underline{x}]$, where $\underline{z}=\left(z_{1}, \ldots, z_{l}\right)$ are $l$ variables over $S$. Assume $V$ has infinite residue field $K$. Let $\underline{\alpha}=\left(\alpha_{1}, \ldots, \alpha_{l}\right)$ be a family of elements of $V$. Let $\widetilde{F}$ be a free 
$\widetilde{S}$-module of finite rank. The specialization $\widetilde{F}_{\underline{\alpha}}$ is a free $S$-module of the same rank. Let $\widetilde{M}$ be a finite $\widetilde{S}$-module and $\phi: \widetilde{F} \rightarrow \widetilde{G} \rightarrow \widetilde{M} \rightarrow 0$ a finite free representation of $\widetilde{M}$ with representing matrix $B=\left(a_{i j}(\underline{z}, \underline{x})\right)$, where $a_{i j}(\underline{z}, \underline{x})=p_{i j}[\underline{z}, \underline{x}] / q[\underline{z}], p_{i j}[\underline{z}, \underline{x}] \in V[\underline{z}, \underline{x}]$ and $q[\underline{z}] \in V[\underline{z}] \backslash \mathfrak{n} V[\underline{z}]$. For any $\underline{\alpha} \in V^{l}$ such that $\overline{q[\underline{\alpha}]} \neq 0$ in $K$, we define $a_{i j}(\underline{\alpha}, \underline{x})=p_{i j}[\underline{\alpha}, \underline{x}] / q[\underline{\alpha}]$ and set $B_{\underline{\alpha}}=\left(a_{i j}(\underline{\alpha}, \underline{x})\right)$. Then $B_{\underline{\alpha}}$ is well defined for almost all $\underline{\alpha}$. The specialization $\phi_{\underline{\alpha}}: \widetilde{F}_{\underline{\alpha}} \rightarrow \widetilde{G}_{\underline{\alpha}}$ of $\phi$ is given by the matrix $B_{\underline{\alpha}}$ provided $B_{\underline{\alpha}}$ is well-defined. The specialization of $\widetilde{M}_{\underline{\alpha}}$ is defined as $\operatorname{coker}\left(\phi_{\underline{\alpha}}\right)$. By [22], $\widetilde{M}_{\underline{\alpha}}$ is well-defined and does not depend on the matrix representation $B$ for almost all $\underline{\alpha}$. It is easy to see that if $M$ is a finite $S$-module and $\widetilde{M}=M \otimes_{S} \widetilde{S}$, then $\widetilde{M}_{\underline{\alpha}} \cong M$. Applying a similar proof of [23], one can show that all the properties of specialization proved in [23] when $V$ is a field also hold if $V$ is a regular local ring.

We arrive at our main theorem. It follows from Propositions 2.4 and 2.2 .

Theorem 2.5. Let $R$ be a Noetherian local ring with infinite residue field, let $I$ be any $R$-ideal, and let $M$ be a finitely generated R-module of dimension $d$. If $x$ is a general element in $I$ then $j_{i}(I, M)=j_{i}(I, M / x M)$ for $0 \leq i \leq d-2$.

Proof. By passing to the completion, we may assume that $R=V / H$, where $(V, \mathfrak{n})$ is a regular local ring. We may further pass to $(V, \mathfrak{n})$ to assume that $(R, \mathfrak{m})$ is a regular local ring. Write $I=\left(a_{1}, \ldots, a_{n}\right)$. Let $\widetilde{R}=R[\underline{z}]_{\mathfrak{m} R[\underline{z}]}$, where $\underline{z}=\left(z_{1}, \ldots, z_{n}\right)$ are $n$ variables over $R$. Let $\widetilde{M}=M \otimes_{R} \widetilde{R}$, $\widetilde{T}=\operatorname{gr}_{I \widetilde{R}}(\widetilde{M})=T \otimes_{R} \widetilde{R}$. Set $\widetilde{x}=\sum_{j=1}^{n} z_{j} a_{j}$ and $\widetilde{T} / \widetilde{x} \widetilde{M}:=\operatorname{gr}_{I \widetilde{R}}(\widetilde{M} / \widetilde{x} \widetilde{M})$. By Proposition 2.4, there exist elements $y_{1}, \ldots, y_{d} \in \mathfrak{m}$ which form an absolutely superficial sequence for both $\widetilde{T}$ and $\widetilde{T} / \widetilde{x} \widetilde{M}$. By Propositions 2.4 and 2.2 if we set $q=\left(y_{1}, \ldots, y_{d}\right)$, then for every $0 \leq i \leq d-2$, we have

$$
(-1)^{i} j_{i}(I, \widetilde{M})=a_{(q, I, \widetilde{M})}^{(1,1)}(0, d-i)=a_{(q, I, \widetilde{M} / \widetilde{x} \widetilde{M})}^{(1,1)}(0, d-i)=(-1)^{i} j_{i}(I, \widetilde{M} / \widetilde{x} \widetilde{M}) .
$$

Furthermore for $1 \leq i \leq d$, set $q_{i}=\left(y_{1}, \ldots, y_{i}\right)$, then

$$
\begin{gathered}
{\left[\left(y_{1}, \ldots, y_{i-1}\right) \widetilde{T}: \widetilde{T} y_{i}\right] \cap\left(y_{1}, \ldots, y_{d}\right) \widetilde{T}=\left(y_{1}, \ldots, y_{i-1}\right) \widetilde{T}} \\
{\left[\left(y_{1}, \ldots, y_{i-1}\right) \widetilde{T} / \widetilde{x} \widetilde{M}: \widetilde{T} / \widetilde{x} \widetilde{M} y_{i}\right] \cap\left(y_{1}, \ldots, y_{d}\right) \widetilde{T} / \widetilde{x} \widetilde{M}=\left(y_{1}, \ldots, y_{i-1}\right) \widetilde{T} / \widetilde{x} \widetilde{M} .}
\end{gathered}
$$

Since $\widetilde{T}$ and $\widetilde{T} / \widetilde{x} \widetilde{M}$ are finite modules over a polynomial ring over the regular local ring $\widetilde{R}$, by [23, 3.6, 3.2], there exists a dense open subset $U$ of $K^{n}$ such that if $\underline{\alpha}=\left(\alpha_{1}, \ldots, \alpha_{n}\right) \in U$, then

$$
\begin{gathered}
{\left[\left(y_{1}, \ldots, y_{i-1}\right) T:_{T} y_{i}\right] \cap\left(y_{1}, \ldots, y_{d}\right) T} \\
=\left(\left[\left(y_{1}, \ldots, y_{i-1}\right) \widetilde{T}:_{\widetilde{T}} y_{i}\right] \cap\left(y_{1}, \ldots, y_{d}\right) \widetilde{T}\right)_{\underline{\alpha}} \\
=\left(\left(y_{1}, \ldots, y_{i-1}\right) \widetilde{T}\right)_{\underline{\alpha}}=\left(y_{1}, \ldots, y_{i-1}\right) T \\
{\left[\left(y_{1}, \ldots, y_{i-1}\right) T / x M:_{T / x M} y_{i}\right] \cap\left(y_{1}, \ldots, y_{d}\right) T / x M} \\
=\left(\left[\left(y_{1}, \ldots, y_{i-1}\right) \widetilde{T} / \widetilde{x} \widetilde{M}: \widetilde{T} / \widetilde{x} \widetilde{M} y_{i}\right] \cap\left(y_{1}, \ldots, y_{d}\right) \widetilde{T} / \widetilde{x} \widetilde{M}\right)_{\underline{\alpha}} \\
=\left(\left(y_{1}, \ldots, y_{i-1}\right) \widetilde{T} / \widetilde{x} \widetilde{M}\right)_{\underline{\alpha}}=\left(y_{1}, \ldots, y_{i-1}\right) T / x M .
\end{gathered}
$$


and the image $\bar{x}=\overline{\sum_{j=1}^{n} \alpha_{j} a_{j}} \in I / q I$ is filter-regular on $T^{\prime}=\operatorname{gr}_{q}(T)$ with respect to the ideal $G_{(01)}^{\prime} G^{\prime}$. Hence

$$
(-1)^{i} j_{i}(I, M)=a_{(q, I, M)}^{(1,1)}(0, d-i)=a_{(q, I, M / x M)}^{(1,1)}(0, d-i)=(-1)^{i} j_{i}(I, M / x M) .
$$

for every $0 \leq i \leq d-2$.

Lemma2.1 also allows us to keep track of the behaviors of $j_{d-1}$ and $j_{d}$ under a general hyperplane section as it has been done for ideals of definition on $M$.

It is well-known that the leading generalized Hilbert coefficient $j_{0}(I, M)$, which is also called the $j$-multiplicity of $I$ on $M$, is preserved under a general hyperplane section (see for instance [12]). The preservation of higher generalized Hilbert coefficients was unknown due to the fact that it is hard to estimate the change of the length of each homogenous component of $\Gamma_{\mathfrak{m}}(T)$ under a general hyperplane section. Our method gives another way to compute the length of homogenous components of $\Gamma_{\mathfrak{m}}(T)$ by using a suitable bigraded module.

As we can see in Example 3.3 under a general hyperplane section, in general only the generalized coefficients $j_{0}, \ldots, j_{d-2}$ are preserved. Indeed, let $S$ be the hypersurface ring obtained by the polynomial ring $R=K[x, y, z, v, w]$ by modding out a general element $\xi$ in the ideal $I=I_{2}(B)$ as defined in Example 3.3. Let $L$ be the ideal $I S$. Notice that $\operatorname{dim} S=3$, the associated graded ring of $L$ is Cohen-Macaulay, however $j_{2}(L)$ is not preserved if we go modulo a general element $v$ in $L$. Notice that $j_{2}(L / v L)=4>1=j_{2}(L)$, i.e. $j_{d-1}$ does not decrease if the initial form of the general element is regular on $G$. This is a general fact that is explained in the next Remark.

Remark 2.6. Assume $R$ has infinite residue field and the grade of $G^{+}$in $G$ is positive. For a general element $x$ in I we have $j_{d-1}(I, M / x M) \geq j_{d-1}(I, M)$.

The above remark follows directly from Proposition 2.2 part (b), as the sum

$$
\sum_{v=2}^{t} \lambda\left(I^{v} M:_{M} x /\left[\left(q^{s+1} I^{v} M+I^{v+1} M\right):_{M} x+I^{v-1} M\right]\right)
$$

vanishes when the initial form of $x$ is regular on $G$.

\section{APPLiCATIONS OF GENERALIZED HiLbERT FUnCTIONS.}

In this section, we are going to discuss the generalized Hilbert function for ideals having minimal or almost minimal $j$-multiplicity on a finite module over a Noetherian local ring.

Let $(R, \mathfrak{m})$ be a Noetherian local ring, let $I$ be an $R$-ideal and let $M$ be a finite $R$-module of $\operatorname{dim} M=d$. Let $G$ and $T$ be defined as before. By adjoining a variable $z$ and passing to the local ring $R[z]_{\mathfrak{m} R[z]}$, we may assume that the residue field of $R$ is infinite. Recall for general elements $x_{1}, \ldots, x_{d}$ in $I$, let $\bar{M}=M /\left(x_{1}, \ldots, x_{d-1}\right) M:_{M} I^{\infty}$. Then $\bar{M}$ is either the zero module or a 1-dimensional CohenMacaulay module and $I$ is an ideal of definition on $\bar{M}$. Then one has the Hilbert-Samuel function of 
I on $\bar{M}$

$$
H_{I, \bar{M}}(t)=\lambda_{\bar{R} / \bar{I}}\left(\bar{M} / I^{t+1} \bar{M}\right)
$$

and the Hilbert-Samuel series of $I$ on $\bar{M}$

$$
h_{I, \bar{M}}(z)=\sum_{t \geq 0} H_{I, \bar{M}}(t) z^{t} .
$$

The following lemma says that the Hilbert-Samuel function $H_{I, \bar{M}}$ does not depend on the general elements $x_{1}, \ldots, x_{d}$ in $I$. The proof follows from the ingredient proved in [13, 2.3], where the independence of $\lambda\left(I \bar{M} / I^{2} \bar{M}\right)$ is established.

Lemma 3.1. Let $R$ be a Noetherian local ring with infinite residue field, let I be any $R$-ideal, and let $M$ be a finitely generated $R$-module of dimension $d$. Then for general elements $x_{1}, \ldots, x_{d}$ in $I$, $\lambda\left(I^{t} \bar{M} / I^{t+1} \bar{M}\right)$ does not depend on $x_{1}, \ldots, x_{d}$ for every $t \geq 0$.

Proof. If $\ell(I, M)<d$ then $\bar{M}=0$. Thus we may assume that $\ell(I, M)=d$. By [13, 2.5], $j(I, M)=$ $j\left(I, I^{t} M\right)$ for every $t \geq 0$. Therefore the case where $t \geq 1$ follows from [13, 2.3]. The case where $t=0$ also follows by a similar argument as in the proof of [13, 2.3].

Recall if $I$ has minimal $j$-multiplicity on $M$, by [17, Theorem 2.9],

$$
h_{I, \bar{M}}(t)=\frac{h_{0}+h_{1} t}{(1-t)^{2}}
$$

where

$$
h_{0}=\lambda(\bar{M} / I \bar{M})=\lambda\left(M /\left[\left(x_{1}, \ldots, x_{d-1}\right) M:_{M} I^{\infty}+I M\right]\right)
$$

and

$$
h_{0}+h_{1}=\lambda\left(I \bar{M} / I^{2} \bar{M}\right)=\lambda\left(I M /\left[\left(x_{1}, \ldots, x_{d-1}\right) M:_{I M} I^{\infty}+I^{2} M\right]\right) .
$$

If $I$ has almost minimal $j$-multiplicity on $M$, by [17, Corollary 4.4],

$$
h_{I, \bar{M}}(t)=\frac{h_{0}+h_{1} t+t^{s}}{(1-t)^{2}}
$$

for some $s \geq 2$, where $h_{0}$ and $h_{1}$ are as above.

Furthermore if $I$ is an ideal of definition on $M$, the Hilbert function is preserved after modding out elements which are regular on the associated graded module $T$. Hence the shape of the HilbertSamuel series of $M$ is the same as the Hilbert-Samuel series of $\bar{M}$. However this is no longer true for arbitrary ideals as the following two examples show.

Example 3.2. Let $R=k[x, y, z, v, w]$ be a polynomial ring over an infinite field $k$ and $I=I_{2}(A)$, where

$$
A=\left(\begin{array}{cccc}
x & y & z & v \\
y & z & v & w
\end{array}\right) .
$$

Observe that I is a perfect ideal of grade 3, I is a complete intersection on the punctured spectrum with analytic spread $\ell(I)=5$. Hence I satisfies $G_{5}$ and $A N_{5}^{-}$. By Macaulay $2, \lambda\left(I^{2} / J I\right)=0$, where $J$ is a general minimal reduction of I. Hence I has minimal j-multiplicity. By [13, 4.9], the associated graded ring $G=\operatorname{gr}_{I}(R)$ is Cohen-Macaulay. 
However by Macaulay 2, the generalized Hilbert-Samuel series of I is:

$$
h_{I}(z)=\frac{z+z^{2}+z^{3}+z^{4}}{(1-z)^{6}}
$$

which does not have the expected shape.

After modding out a general element $\xi_{1}$, two general elements $\xi_{1}, \xi_{2}$, three general elements $\xi_{1}, \xi_{2}, \xi_{3}$, and four general elements $\xi_{1}, \xi_{2}, \xi_{3}, \xi_{4}$ in I, the generalized Hilbert-Samuel series are $\frac{z+z^{2}+z^{3}+z^{4}}{(1-z)^{5}}, \frac{z+z^{2}+z^{3}+z^{4}}{(1-z)^{4}}, \frac{z+z^{2}+z^{3}+z^{4}}{(1-z)^{3}}$ and $\frac{4 z}{(1-z)^{2}}$ respectively. The Hilbert-Samuel series modulo the residual intersection $\left(\xi_{1}, \xi_{2}, \xi_{3}, \xi_{4}\right): I^{\infty}$ is $h_{\bar{I}}(z)=\frac{3+z}{(1-z)^{2}}$.

In the previous example the generalized Hilbert series was preserved until the height of the ideal was reduced to zero. One could imagine that this is always true if $G$ is Cohen-Macaulay. However the next example shows that the Cohen-Macaulayness of $G$ does not suffice to preserve the generalized Hilbert-Samuel series:

Example 3.3. Let $R=k[x, y, z, v]$ be a polynomial ring over an infinite field $k$ and $I=I_{2}(B)$, where

$$
B=\left(\begin{array}{llll}
x & y & z & v \\
v & x & y & z
\end{array}\right)
$$

Observe that I is a perfect ideal of grade 3, I is a generically a complete intersection with analytic spread $\ell(I)=4$. Hence I satisfies $G_{4}$ and $A N_{4}^{-}$. Let $J$ be a general minimal reduction of $I$, using Macaulay 2, one computes $\lambda\left(I^{2} / J I\right)=1$. Hence I has almost minimal j-multiplicity. By [13, 4.9], the associated graded ring $G=\operatorname{gr}_{I}(R)$ has depth at least 3. Indeed, $G$ is Cohen-Macaulay.

However by Macaulay 2, the generalized Hilbert-Samuel series of I is:

$$
h_{I}(z)=\frac{4 z+z^{2}+6 z^{3}-3 z^{4}}{(1-z)^{5}}
$$

which does not have the expected shape.

After modding out a general element $\xi_{1}$, two general elements $\xi_{1}, \xi_{2}$, and three general elements $\xi_{1}, \xi_{2}, \xi_{3}$ in I, the generalized Hilbert-Samuel series are $\frac{4 z+z^{2}+6 z^{3}-3 z^{4}}{(1-z)^{4}}, \frac{4 z+4 z^{2}}{(1-z)^{3}}$ and $\frac{7 z+z^{2}}{(1-z)^{2}}$ respectively. The Hilbert-Samuel series modulo the residual intersection $\left(\xi_{1}, \xi_{2}, \xi_{3}\right): I^{\infty}$ is $h_{\bar{I}}(z)=\frac{1+6 z+z^{2}}{(1-z)^{2}}$.

Finally we provide a sufficient condition to ensure that the generalized Hilbert-Samuel series of ideals having minimal or almost minimal multiplicity has the desired shape.

Theorem 3.4. Let $M$ be a Cohen-Macaulay module of dimension d over a Noetherian local ring $(R, \mathfrak{m})$ and let $I$ be an $R$-ideal with $\ell(I, M)=d$. Assume depth $(M / I M) \geq 1$ and I satisfies $G_{d}$ and $A N_{d-2}^{-}$on $M$. Let $q \subseteq \mathfrak{m}$ be an ideal of definition on $M / I M$ that is generated by a d-sequence on the associated graded module $T$. Let $x_{1}, \ldots, x_{d}$ be general elements in I and set $J_{i}=\left(x_{1}, \ldots, x_{i}\right)$ for $1 \leq i \leq d$.

(a) Assume I has minimal $j$-multiplicity and for $1 \leq i \leq d-1$ and $s>>0$,

$$
J_{i} I M \cap\left(q^{s+1} I I M+I^{2} I M\right)=J_{i}\left(q^{s+1} I M+I^{2} M\right),
$$


then $h_{I, M}(z)=\frac{\varepsilon_{0} z+\varepsilon_{1} z^{2}}{(1-z)^{d+1}}$, where $\varepsilon_{0}=\lambda\left(\Gamma_{\mathfrak{m}}\left(I M / I^{2} M\right)\right)$ and $\varepsilon_{1}=h_{0}+h_{1}-\varepsilon_{0}$ with $h_{0}+h_{1}=$ $\lambda\left(I \bar{M} / I^{2} \bar{M}\right)=\lambda\left(I M /\left(x_{1}, \ldots, x_{d-1}\right) M+I^{2} M\right)$ defined above.

(b) Assume I has almost minimal j-multiplicity and for $1 \leq t \leq r(I, M), 1 \leq i \leq d-1$ and $s>>0$,

$$
J_{i} I^{t-1} I M \cap\left(q^{s+1} I^{t} I M+I^{t+1} I M\right)=J_{i}\left(q^{s+1} I^{t-1} I M+I^{t} I M\right),
$$

then $h_{I, M}(z)=\frac{\varepsilon_{0} z+\varepsilon_{1} z^{2}+z^{s}}{(1-z)^{d+1}}$ for some integer $s \geq 3$, where $\varepsilon_{0}$ and $\varepsilon_{1}$ are the same as in part (a).

Proof. By [13], $T$ is Cohen-Macaulay (respectively, almost Cohen-Macaulay) if $I$ has minimal (respectively, almost minimal) $j$-multiplicity. Furthermore the initial forms $x_{1}^{*}, \ldots, x_{d-1}^{*}$ in $G$ form a regular sequence on $T_{+}$. Hence $I^{t} I M:_{I M} x=I^{t-1} I M$ for every $t \geq 1$. Appling Lemma 2.1 to the module $I M$, one has for every $s, t \geq 0$,

$$
\begin{aligned}
& H_{(q, I, I M)}^{(1,0)}(s, t)=H_{\left(q, I, I M / x_{1} I M\right)}^{(1, I)}(s, t) \\
& -\sum_{v=1}^{t} \lambda\left(\left[\left(q^{s+1} I^{V} I M+I^{v+1} I M\right):_{I^{V-1} I M} x\right] /\left[q^{s+1} I^{v-1} I M+I^{v} I M\right]\right) .
\end{aligned}
$$

By [13], $r(I, M)=1$ in part (a). Applying a similar argument as in [5], one can show that for $s>>0$, the images of $x_{1}, \ldots, x_{d-1}$ in $T_{+} / q^{s+1} T_{+}$form a regular sequence on $T_{+} / q^{s+1} T_{+}$. Hence $\left(q^{s+1} I^{t} I M+I^{t+1} I M\right):_{I^{t-1} I M} x=q^{s+1} I^{t-1} I M+I^{t} I M$ for every $t \geq 1$. Therefore $H_{(q, I, I M)}^{(1,0)}(s, t)=$ $H_{\left(q, I, I M / x_{1} I M\right)}^{(1, I)}(s, t)$ for every $s, t \geq 0$. This shows that the h-polynomial of the generalized HilbertSamuel series of $I$ on $I M$ are preserved after modulo $x_{1}, \ldots, x_{d-1}$. Since $\Gamma_{0}(M / I M)=0$, one has $H_{(q, I, M)}^{(1,0)}(s, t)=H_{(q, I, I M)}^{(1,0)}(s, t-1)$ and $h_{I, M}(z)=h_{I, I M}(z) z$. After lifting back the Hilbert-Samuel series in the 1-dimensional case, we obtain the desired result.

\section{REFERENCES}

[1] S. Abhyankar, Local rings of high embedding dimension, Amer. J. Math. 89 (1967), 1073-1077.

[2] R. Achilles and M. Manaresi, Multiplicity for ideals of maximal analytic spread and intersection theory, J. Math. Kyoto Univ. 33-4 (1993) 1029-1046.

[3] R. Achilles and M. Manaresi, Multiplicities of a bigraded ring and intersection theory, Math. Ann. 309 (1997), 573-591.

[4] C. Ciupercă, A numerical characterization of the $S_{2}$-ification of a Rees algebra, J. Pure and Applied Algebra, 178 (2003), 25-48

[5] A. Corso, L. Ghezzi, C. Polini, and Bernd Ulrich, Cohen-Macaulayness of special fiber rings, Communications in Algebra, 31 (2003), 3713-3734.

[6] A. Corso, C. Polini, and M. Vaz Pinto, Sally modules and associated graded rings, Communications in Algebra, 26 (1998), 2689-2708.

[7] J. Elias, On the depth of the tangent cone and the growth of the Hilbert function, Trans. Amer. Math. Soc., 351 (1999), 4027-4042.

[8] S. Huckaba, On the associated graded rings having almost maximal depth, Comm. Algebra, 26 (1998), 967-976.

[9] C. Huneke, On the symmetric and Rees algebra of an ideal generated by a $d$-sequence, J. Algebra, 62 (1980), 268-275.

[10] P. Mantero and Y. Xie, Generalized stretched ideals and Sally's conjecture, Submitted.

[11] G. Colome Nin, C. Polini, B. Ulrich and Y. Xie, Normalizations of arbitrary ideals, in progress.

[12] K. Nishida and B. Ulrich, Computing $j$-multiplicities, to appear in J. Pure Appl. Algebra.

[13] C. Polini and Y. Xie, $j$-multiplicity and depth of associated graded modules, Submitted. 
[14] T. Puthenpurakal, Ratliff-Rush filtration, regularity and depth of higher associated graded modules, Part I, J. Pure Applied Algebra, 208 (2007), 159-176.

[15] M. Rossi and G. Valla, A conjecture of J. Sally, Communications in Algebra, 24 (13) (1996), 4249-4261.

[16] M. Rossi and G. Valla, Cohen-Macaulay rings of dimension two and an extended version of a conjecture of J. Sally, J. Pure Appl. Algebra, 122 (1997), 293-311.

[17] M. Rossi and G. Valla, Hilbert functions of filtered modules, Lecture Notes of the Unione Matematica Italiana, 9. Springer-Verlag, Berlin, UMI, Bologna, 2010.

[18] M. Rossi, Primary ideals with good associated graded rings, J. Pure Appl. Algebra, 145 (2000), 75-90.

[19] J. Sally, On the associated graded ring of a local Cohen-Macaulay ring, J. Math. Kyoto Univ. 17 (1977), no. 1, 19-21.

[20] C. Huneke and I. Swanson, Integral closure of ideals, rings, and modules, London Mathematical Society Lecture Note Series 336, Cambridge University Press, Cambridge, 2006.

[21] N. V. Trung, Absolutely superficial sequences, Math. Proc. Camb. Phil. Soc. 93 (1983), 35-47.

[22] N. V. Trung, Constructive characterization of the reduction numbers, Compositio Math. 137 (2003), 99-113.

[23] N. V. Trung, Specialization of modules, Communications in Algebra 27(6) (1999), 2959-2978.

[24] H. J. Wang, On Cohen-Macaulay local rings with embedding dimension e+d-2, J. Algebra 190 (1997), no.1, 226240.

[25] Y. Xie, Formulas for the multiplicity of graded algebras, to appear in Trans. Amer. Math. Soc.

Department of Mathematics, University of Notre Dame, Notre Dame, Indiana 46556

E-mail address: cpolini@nd.edu

Department of Mathematics,University of Notre Dame, Notre Dame, Indiana 46556

E-mail address: yxie@nd.edu 